wirken sich nur auf die ärztlichen Verordnungen aus. Ein Honorar ist leider nicht vorgesehen - eine Schlechterstellung gegenüber der $S A P V$, wo Erst- und Folgeverordnungen nach den Nrn. 01425 und 01426 EBM abgerechnet werden können und immerhin 26,64 bzw.

16,01 Euro einbringen (Tab. 1).

Für die Verordnung häuslicher Krankenpflege gibt es das Formular 12, auf dem die neue Leistungsziffer 24a vermerkt werden muss. Unabhängig davon können alle notwendigen behandlungspflegerischen Maßnahmen, die zum Zeitpunkt der Verordnung bekannt sind und im Rahmen der Nr. 24a erbracht werden, wie gewohnt auf der Verordnung angegeben werden. Eine gesonderte Qualifikation ist nicht notwendig.

Neu ist auch die Regelung zur Medikamentengabe nach Nr. 26 im Leistungsverzeichnis der HKP-Richtlinie. Die Leistungsbeschreibung wird geändert, um klarzustellen, dass

Tab. 1 Extrabudgetär vergütete Leistungen für die Verordnung der spezialisierten ambulanten Palliativversorgung (SAPV)

\begin{tabular}{|l|l|l|}
\hline EBM & Leistungsbeschreibung & Euro \\
\hline 01425 & Erstverordnung der SAPV & 26,64 \\
\hline 01426 & Folgeverordnung der SAPV & 16,01 \\
\hline 03370 & $\begin{array}{l}\text { Palliativmedizinische Ersterhebung des Patientenstatus inklusive } \\
\text { Behandlungsplan (einmal im Krankheitsfall) }\end{array}$ & 35,91 \\
\hline 03371 & $\begin{array}{l}\text { Zuschlag zur Versichertenpauschale für die palliativmedizinische } \\
\text { Betreuung in der Arztpraxis (einmal im Krankheitsfall) }\end{array}$ & 16,74 \\
\hline 03372 & $\begin{array}{l}\text { Zuschlag zu den Besuchsziffern 01410 und 01413 für die palliativ- } \\
\text { medizinische Betreuung in der Häuslichkeit (je vollendete 15 Minuten) }\end{array}$ & 13,06 \\
\hline 03373 & $\begin{array}{l}\text { Zuschlag zu den Besuchsziffern 01411, 01412 und 01415 für die palliativ- } \\
\text { medizinische Betreuung in der Häuslichkeit (je Besuch) }\end{array}$ & 13,06 \\
\hline
\end{tabular}

die Medikamentengabe einerseits das Richten und andererseits auch das Verabreichen umfasst. Das Verabreichen von ärztlich ver- ordneten Medikamenten beinhaltet auch die notwendige Vorbereitung, u. a. die Dosierung der Darreichungsform.

\title{
Nach dem Aus für den Guajaktest: Welche Praxis-Testverfahren bleiben uns?
}

_ Seit dem 1. April 2017 gibt es eine neue Abrechnungsposition im Rahmen der Stuhldiagnostik bei der Krebsvorsorge. Mit der Nr. 01737 EBM kann dann der neue quantitative immunologische Test (iFOBT) berechnet werden. Er ist mit 6 Euro extrabudgetär bewertet (siehe MMW 5/2017, S. 25). Die Nr. 01734 für den guajakbasierten Test ist ebenso entfallen wie die Nr. 40150 für die Ausgabe von drei Testbriefchen ohne Probenanalyse.

In kurativen Fällen gibt es eine Übergangsregelung: Noch bis zum 1. Oktober 2017 kann der Guajaktest mit der Nr. 32040 abgerechnet werden. Danach kommt auch hier ausschließlich der iFOBT zum Einsatz - abrechnen kann man ihn dann allerdings nicht mehr.

\section{MMW-KOMMENTAR}

Der Stuhltest wird in der Hausarztpraxis also nicht mehr analysiert - aber man sollte nicht vergessen, dass es andere einfache Testmethoden gibt, die man auch weiterhin in der Praxis durchführen und abrechnen kann.

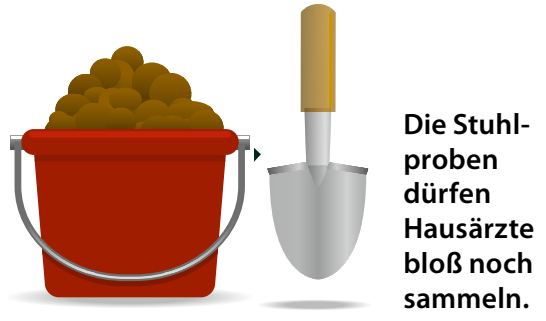

Die Nr. 02400 steht für die Durchführung des ${ }^{13} \mathrm{C}$-Harnstoff-Atemtests ohne analytische Auswertung und ist mit 2,42 Euro bewertet. Die Leistung ist allerdings nur zur Erfolgskontrolle nach Eradikationstherapie einer Helicobacter-pylori-Infektion berechnungsfähig. Nach Ende der Therapie müssen mindestens vier Wochen vergangen sein. Auch bei Kindern mit begründetem Verdacht auf eine Ulkuserkrankung kann der Test gemacht werden.

Die Verordnung des ${ }^{13} \mathrm{C}$-Harnstoffs als Fertigpräparat ist je nach Vertragslage über Sprechstundenbedarf oder auf den Namen des Patienten möglich. Bei Bezug der notwendigen Materialien durch den Arzt können die entstandenen Kosten pauschal nach
$\mathrm{Nr} .40154$ berechnet werden. Auch den $\mathrm{H}_{2}-$ Atemtest sollte man nicht vergessen. Er wird mit der Nr. 02401 abgerechnet. Die Nr. ist mit 11,37 Euro bewertet. Der Test dient der Diagnose verschiedener Darmerkrankungen, z. B. der Laktose- oder der Fruktoseintoleranz. Sofern mehrere Atemtests mit unterschiedlichen Kohlehydraten erforderlich werden, kann die Nr. 02401 entsprechend der Anzahl der Testsubstanzen berechnet werden. Die Kosten der Testsubstanz sind mit der Gebühr abgegolten und daher nicht besonders berechnungsfähig.

Dr. Gerd W. Zimmermann, Hofheim

\section{Kleine Korrektur}

In Ausgabe 5/2017, S. 25, hat sich in unsere Tabelle zum Vorsorgespektrum bei männlichen Patienten in der Hausarztpraxis ein kleiner Fehler eingeschlichen. Die Ausgabe des neuen iFOBT wird nicht mit der $\mathrm{Nr}$. 01735 berechnet, sondern - wie es auch im Text steht - mit der Nr. 01737. 